\title{
Tunable diode laser spectroscopy with optimum wavelength scanning
}

\author{
J. Chen • A. Hangauer · R. Strzoda • M.-C. Amann
}

Received: 15 October 2009 / Revised version: 15 February 2010 / Published online: 25 March 2010

(c) Springer-Verlag 2010

\begin{abstract}
In this paper, an improved signal processing method is given, aiming at a sensitivity improvement of the tunable diode laser spectroscopy based gas concentration measurement. The gas absorption spectrum is probed with an optimum wavelength scanning function which enables a more efficient curve fit than for traditional linear wavelength scanning. The wavelength scanning function is determined to be optimum, in the sense that the variance of the estimated concentration noise is minimized. This optimum scanning function depends on the signal model used in curve fitting. Several models including interfering gas spectra and etalon fringes are examined. Compared to the gas absorption spectrum recorded by ramping the wavelength linearly, the optimum spectrum waveforms have a cascade structure, which means that the optimum scanning preferably samples important points of the spectrum. The new method theoretically enables a factor of $\sim 2$ improvement on detection sensitivity of the estimated concentration. Furthermore, direct spectroscopy is superior to second harmonic detection, because the concentration noise can be an additional factor of $\sim 2$ lower.
\end{abstract}

\section{Introduction}

Tunable diode laser spectroscopy (TDLS) detects gas concentration by taking a spectral fingerprint of the gas absorption. The gas absorption spectrum $A(\lambda)$ is given by the quo-

J. Chen $(\varangle) \cdot$ A. Hangauer · M.-C. Amann

Walter Schottky Institut, Technische Universität München, Am Coulombwall 3, 85748 Garching, Germany

e-mail: jia.chen@wsi.tum.de

J. Chen $\cdot$ A. Hangauer $\cdot$ R. Strzoda

Corporate Research and Technologies, Siemens AG,

Otto-Hahn-Ring 6, 80200 Munich, Germany tient of ingoing $\left(I_{0}\right)$ and outgoing $(I)$ laser light intensities through a gas sample:

$A(\lambda)=\frac{I_{0}(\lambda)-I(\lambda)}{I_{0}(\lambda)}$.

Usually, in the sensor the gas absorption is measured directly, e.g. by implementing (1) (direct spectroscopy), or indirectly by using derivative spectroscopy, e.g. wavelength modulation spectroscopy (WMS) [1].

A common task of a TDLS-based gas sensor is to achieve high sensing sensitivity. To improve the sensing sensitivity of a sensor the following basic methods exist:

- Probe a stronger absorption line, e.g. using lines in the fundamental gas absorption band in the mid-infrared range.

- Increase the optical path length, e.g. by using multi-pass cells or cavity-enhanced methods, e.g. Integrated Cavity Output Spectroscopy (ICOS) [2, 3].

- Lower the noise induced by laser and detector, e.g. by applying a low-noise laser and/or using a higher laser power to improve the detector signal to noise ratio, in the case laser noise is not dominating. In the other case, laser noise compensation methods can also be employed, e.g. balanced detection [4, 5].

- Average the concentration values over time to reduce the overall sensor bandwidth, which lowers the sensor noise level. This method corresponds to application of a filter with rectangular impulse response. More sophisticated methods employ time-variant filters with an adaptive filtering bandwidth like the Kalman filter [6-8].

- Use optimized signal processing, e.g. linear least squares curve fitting. At a given sensor bandwidth linear least squares curve fitting is the best linear method to filter the measurement noise, because it gives the smallest variance 
on the fitted concentration values among all linear digital filtering methods, e.g. low pass/high pass or Wiener filtering $[9,10]$. However, the performance of curve fitting depends on the signal model for the measured data. Therefore, the sensor performance can be improved if the measurement data is recorded in an adjusted way and the corresponding signal model is changed accordingly. This method is discussed in the following sections.

Usually, the laser wavelength is linearly ramped with respect to time to scan the absorption, so a linear wavelength scanning function ${ }^{1}$ as shown in Fig. 1a is used. The recorded spectrum waveform ${ }^{2}$ is disregarding the $x$-axis unit $(t$ instead of $\lambda$ ) equal to the gas absorption spectrum. This is shown in Fig. 1b for direct spectroscopy [11] and Fig. 1c for WMS second harmonic [1], respectively. After each spectral scan, a curve fit is applied to determine the gas concentration, which then is an output value of the sensor (Fig. 2).

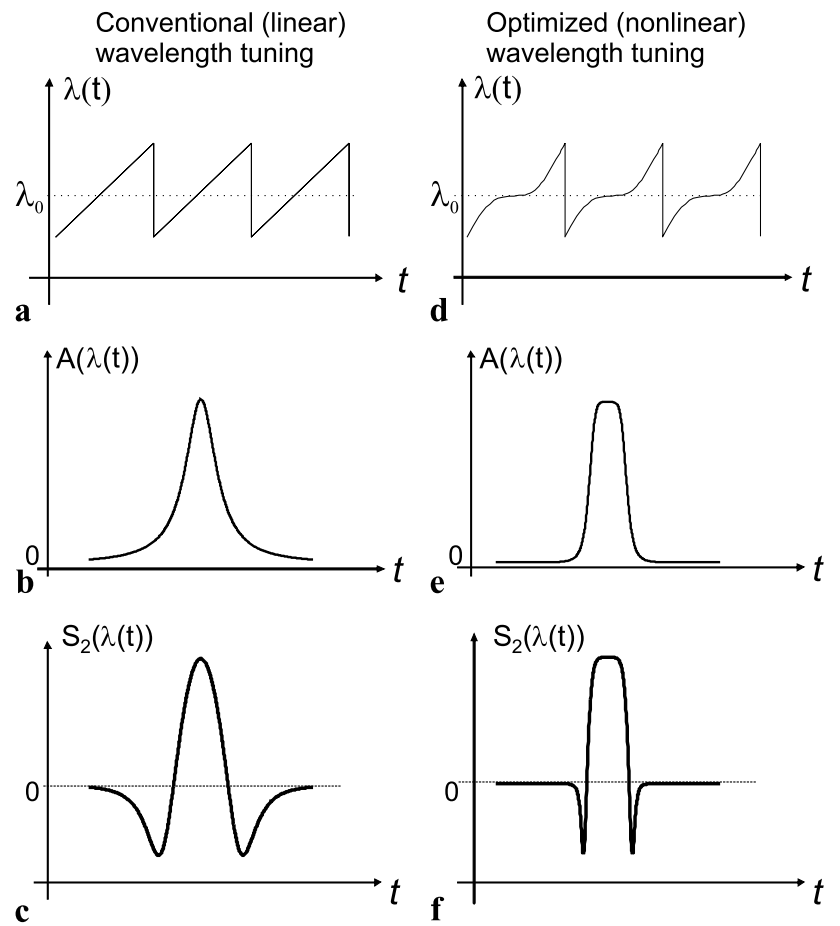

Fig. 1 Schematic illustration of the wavelength scanning function: conventional (linear) (a) and optimized (nonlinear) (d). The resulting spectrum waveform is shown for direct spectroscopy in (b) for linear scanning and (e) for nonlinear scanning as well as for WMS (second harmonic detection) in (c) and (f)

\footnotetext{
${ }^{1}$ The wavelength scanning function is the function describing the laser emission wavelength over time. The wavelength scanning function is applied to scan the gas absorption spectrum.

${ }^{2}$ The spectrum waveform is the signal obtained by scanning the gas absorption spectrum over time with a given wavelength scanning function.
}

However, with linear laser wavelength scanning the signal-to-noise ratio (SNR) on the estimated gas concentration may not be optimum. The SNR is defined as the averaged sensor output values versus their standard deviation at constant gas concentration. The variation of the sensor output originates from the measurement noise on the spectrum that 'passes through' the sensor signal processing, i.e. curve fitting. Basically, if a single line is probed, the gas absorption at center wavelength carries the highest information content of the gas concentration and thus should be sampled more often than the other spectral ranges. This is nonlinear wavelength sampling because the wavelength scanning function over time is nonlinear. An example is shown in Fig. 1d; the resulting spectrum waveform is shown in Fig. 1e and for direct spectroscopy and WMS, which are more flat at the center wavelength, because more time is spent to sample the center wavelength. A special case of nonlinear scanning is applied in [12]. The wavelength scanning jumps over the spectral range where no absorption line is present and therefore optimizes scanning of two separate absorption lines. In the following, this approach is generalized and the optimum scanning method and corresponding SNR improvement is determined.

Whether a sensor is limited by white noise or slow drifts depends on the measurement rate of the sensor. At high measurement rate (low averaging time) the limitation is always given by white noise, as commonly seen on the characteristic slope of -1 in a typical Allan plot [13]. However, sensors for trace-gas analysis are often operated at their optimum averaging time (minimum of the Allan plot) where the sensor resolution is given by other noise sources than white noise, e.g. drifts or $1 / f$ noise $[14,15]$. White noise is always present (e.g. detector shot noise or amplifier thermal noise) and $1 / f$ noise and drift have very different sources which strongly depend on the electrical and mechanical components. Furthermore, the contribution of $1 / f$ noise generated in the detector or amplifier electronics is generally suppressed by technical measures, e.g. detection of a harmonic component in the $\mathrm{kHz}$ range or direct spectroscopy with scanning rates in the $\mathrm{kHz}$ range. In this paper only the white noise contribution of sensor noise is considered, which is the dominating noise source for sensors whose averaging time is well below the minimum of the Allan plot. The aim of this paper is to analyze the optimum case, i.e. to determine the upper limit of improvement that is possible to achieve by nonlinear scanning. This method may reduce the optimum averaging time (minimum of the Allan plot), because the white noise suppression is improved. For white noise limited systems (high measurement rate) the concentration resolution is enhanced. The nonlinear scanning method thus improves either sensor response time (for drift or $1 / f$ noise limited systems) or concentration resolution (for white noise limited systems). 


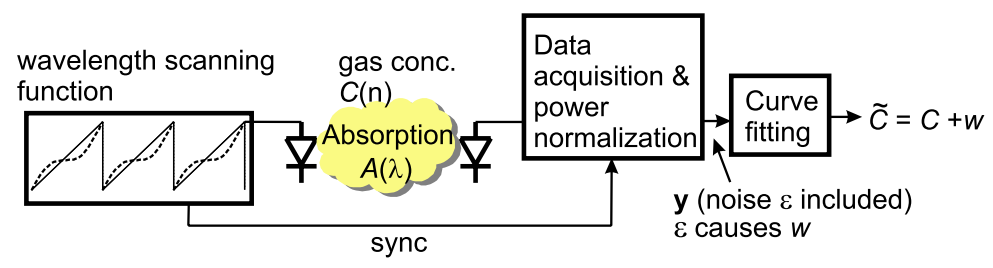

Fig. 2 Sensing block diagram: the laser is tuned with the wavelength scanning function: linear (solid curve) or nonlinear (broken curve). After power normalization the measured data set $\boldsymbol{y}$ is a pure measure of the gas absorption spectrum, and concentration estimation is done

To maximize the SNR on the concentration values, the spectrum waveform has to be chosen properly. Basically, the spectrum waveform can be varied by:

- Scanning the laser current nonlinearly with respect to time so that certain wavelength ranges containing more information of the gas concentration are sampled more often than the less important wavelength ranges. The gas spectrum is not necessarily sampled at equally spaced wavelength points.

- Varying the modulation index $m=\lambda_{a} / a_{L}$ if WMS is applied. Here $\lambda_{a}$ is the amplitude of the sinusoidal modulation in wavelength and $a_{L}$ the half width at half maximum (HWHM) of the absorption line. $m$ is typically set to a value aiming at the highest signal peak, which is around 2.2 for second harmonic detection for the Lorentzian gas profile [1]. But, it is not necessarily optimum in terms of minimal concentration noise because the scanned spectrum also contains other points, e.g. minima, which have then possibly lower signal amplitude and overcompensate the high center peak.

- Varying the wavelength range which is scanned. If the spectral baseline, i.e. slope, offset or parabolic terms, is unknown or the target gas absorption is interfered with other gas absorptions, a larger spectral range than only the absorption peak value should be scanned.

We present in this paper a universal criterion to determine the optimum spectrum waveform and thus also the corresponding wavelength scanning function. This includes how broad the scanned spectral range should be andif WMS is applied-the optimum modulation index $m$. The optimum spectrum waveform depends on the signal model.

In most cases, the more components are included in the signal model and the more parameters are fitted, the less gain in SNR on the estimated target gas concentration value by the curve fit is achieved. No degradation in signal to noise ratio is observed if the additional components are orthogonal to the existing ones. Usually, the measured signal consists of several components, e.g. the spectral components belonging to the target gas absorption, the absorption spectrum of by a curve fit. $\epsilon$ represents the inevitable noise induced by the measurement apparatus, which is included in $\boldsymbol{y}$. The noise $w$ on the estimated concentration values $\tilde{C}$ originates only from measurement noise $\boldsymbol{\epsilon}$

interfering gases, the spectral baseline consisting of offset, linear or parabolic terms (caused by the laser residual amplitude modulation (RAM) [16]) and etalon fringes caused by unwanted optical interference. These components for the measured data are used in the curve fit and form the 'signal model' which is needed for every curve fit. The components can be of both theoretical and experimental origin. Components which are quantitatively known from a reference measurement or a theoretical calculation are not included in the curve-fit model, because they are subtracted before curve fitting. One approach is described in [14], where repeated background measurements are performed and the determined background is subtracted from measurement data before the spectrum curve fitting. In this case the curve-fit model can contain additional components like offset or slope to compensate for incomplete background subtraction [15]. Another approach with an adaptive curve-fit model which includes several possible background patterns is described in [17]. Different signal models for the measurement data can be distinguished by the a priori information which is available in the specific situation. The 'value' of the a priori information can be determined, because different models give different SNR improvements in the curve fit. For example, it turns out that by knowing the spectral baseline the sensor resolution can theoretically be a factor of two better than without the knowledge of the spectral baseline (see Table 1).

In this paper, three signal models are discussed: (I) only the gas concentration is fitted (spectral baseline and spectral components and concentrations of interfering gases are known), (II) the target gas concentration and linear baseline are fitted and (III) the concentrations of target gas and interfering gas, spectral baseline and the amplitudes and phases of etalon fringes with known period lengths are fitted. We derive the optimum wavelength scanning function analytically for models I and II and numerically for model III. The solution for a linear model is also applicable for nonlinear models, by linearizing it with a Taylor series. This is discussed in Sect. 2.7. 


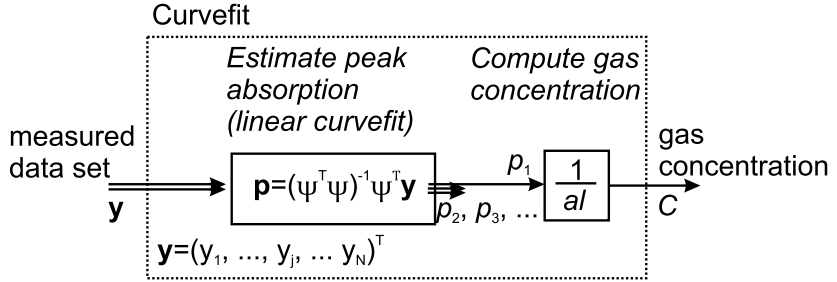

Fig. 3 The estimated gas concentration is the linear least squares fit solution of the measured data $\boldsymbol{y}$ divided by $a \cdot l$

\section{Determination of the optimum spectrum waveform and scanning wavelength function}

\subsection{Optimization criterion}

In Fig. 2, it is shown that the laser diode is tuned with a linear or nonlinear wavelength scanning function. The gas absorption spectrum recorded at the photodiode is powernormalized and is a pure measure of the gas absorption. A curve fit is carried out on the sampled measurement data $\boldsymbol{y}$ to estimate the gas concentration, whereas $\boldsymbol{\epsilon}$ represents the noise of $\boldsymbol{y}$ induced by the measurement system, i.e. laser noise, shot noise, thermal noise and electronics noise. The curve-fit result $\tilde{C}$ is the true gas concentration $C$ plus noise $w$, which originates from $\epsilon$. So, we have

$\tilde{C}=C+w$,

$\sigma_{w}^{2}=\left\langle w^{2}\right\rangle$

with \langle\rangle denoting the expectation value. To investigate the optimum spectrum waveform or the optimum wavelength scanning function the noise variance $\sigma_{w}^{2}$ is minimized. The sensor sensitivity (minimum detectable concentration) is equal to $\sigma_{w}$ and is thus optimum. In the following, we derive an analytical expression for $\sigma_{w}^{2}$.

\subsection{Signal model of curve fit}

The vector $\boldsymbol{y}$ represents the measured data and is the sum of the sampled direct absorption spectrum or harmonic spectrum, spectral baseline, measurement noise, etc. It consists of $N$ data points. The data sample rate is $R N$, with $R$ (unit: $\mathrm{Hz}$ ) being the measurement rate, i.e. the rate with which spectra are scanned and concentration values are output.

The vector $\boldsymbol{y}$ can be expressed by the following model:

$\boldsymbol{y}=\underbrace{\left(\boldsymbol{\psi}_{1}\left|\boldsymbol{\psi}_{2}\right| \cdots\right)}_{\boldsymbol{\Psi}}\left(\begin{array}{c}p_{1} \\ p_{2} \\ \vdots\end{array}\right)+\boldsymbol{\epsilon}$.

$\boldsymbol{\psi}_{1}, \boldsymbol{\psi}_{2}, \ldots$ are the column vectors of the model matrix $\boldsymbol{\Psi}$, which represent the spectra components linearly weighted

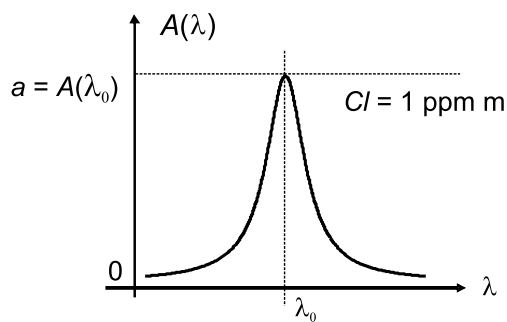

Fig. 4 Schematic explanation of the peak absorption coefficient $a$ : it is the amplitude of a $1 \mathrm{ppm} \mathrm{m}$ gas absorption spectrum

with the parameters $p_{1}, p_{2}, \ldots$ and superimposed with noise $\boldsymbol{\epsilon}$ to form the measured spectrum $\boldsymbol{y}$.

The parameters $p_{i}$ are for example the peak absorption values of the target or interfering gases that are present in the optical cell; offset; slope and amplitude, phase of etalon fringes. $p_{1}$ is the peak gas absorption value of the desired gas, which is the product of gas concentration $C$, optical path length $l$ and peak absorption coefficient $a$ (see Fig. 4):

$a=\left.A\left(\lambda_{0}\right)\right|_{C \cdot l=1 \mathrm{ppm} \mathrm{m}}$,

where $\lambda_{0}$ is the wavelength of the absorption line center orin the case of a complex spectrum consisting of many overlapping lines of one gas - the wavelength of the peak value. The gas concentration $C$ is estimated from $\boldsymbol{y}$ by a linear least squares curve fit [18] and has the following analytical expression:

$C=\frac{p_{1}}{a l}=\frac{1}{a l}\left(\left(\boldsymbol{\Psi}^{T} \boldsymbol{\Psi}\right)^{-1} \boldsymbol{\Psi}^{T} \boldsymbol{y}\right)_{1}$,

where the subscript notation $(\boldsymbol{X})_{1}$ denotes the first element of the vector $\boldsymbol{X}$. The curve-fit process is schematically explained in Fig. 3.

The $\psi_{i}$ are the spectrum waveforms of the target gas and interfering gases, the spectral background (offset, slope) and etalon fringes. The first column $\psi_{1}$ is the spectrum waveform of the target gas and has the following expression for direct spectroscopy:

$\psi_{1}=\left(\frac{A\left(\lambda_{1}\right)}{a C l}, \frac{A\left(\lambda_{2}\right)}{a C l}, \ldots, \frac{A\left(\lambda_{N}\right)}{a C l}\right)^{T}$

with the vector of sampled wavelength points:

$\lambda=\left(\lambda_{1}, \lambda_{2}, \ldots, \lambda_{N}\right)^{T}$.

For WMS with second harmonic detection it is given by

$\psi_{1}=\left(\frac{S_{2}\left(\lambda_{1}\right)}{a C l}, \frac{S_{2}\left(\lambda_{2}\right)}{a C l}, \ldots, \frac{S_{2}\left(\lambda_{N}\right)}{a C l}\right)^{T}$

$\psi_{1}$ is independent of which gas is investigated, the gas concentration $C$ as well as the optical path length $l$. In the case 


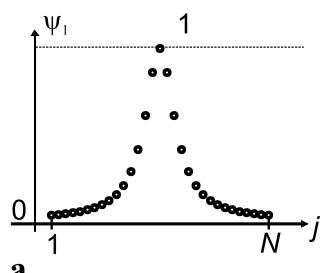

$\mathbf{a}$

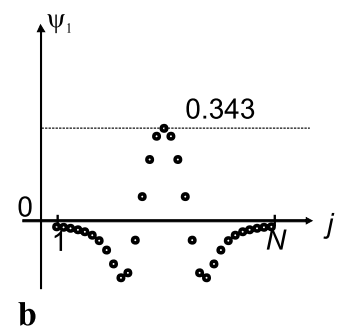

Fig. 5 Examples of sampled spectrum waveforms $\boldsymbol{\psi}_{1}$ for linear scanning in (a) direct spectroscopy and (b) WMS (second harmonic) are shown

of WMS the definition of the second harmonic spectrum $S_{2}(\lambda)$ is taken from [19]:

$S_{2}(\lambda)=-\frac{1}{\pi} \int_{-\pi}^{\pi} A\left(\lambda+\lambda_{a} \cos (z)\right) \cos (2 z) \mathrm{d} z$,

with $\lambda_{a}$ being the amplitude of wavelength modulation. $\psi_{1}$ has a maximum value of 1 for direct spectroscopy and is less than 1 for WMS. Examples of linearly scanned $\psi_{1}$ in direct spectroscopy and WMS (second harmonic) are shown in Fig. 5a and b, respectively.

\subsection{Analytical expression for noise variance $\sigma_{w}^{2}$}

As explained before, the measurement noise $\epsilon$ is assumed to be white noise, i.e.

$\langle\boldsymbol{\epsilon}\rangle=\mathbf{0}, \quad\left\langle\boldsymbol{\epsilon} \cdot \boldsymbol{\epsilon}^{T}\right\rangle=\sigma^{2} \boldsymbol{I}$,

with $\boldsymbol{I}$ being the identity matrix. The noise variance $\sigma^{2}$ is expressed by the product of noise power density $S_{y y}$ on measured data $\boldsymbol{y}$ and the noise bandwidth $R N$ :

$\sigma^{2}=S_{y y} R N$

The relationship between $\sigma^{2}$ and $\sigma_{w}^{2}$ is given by taking the variance of (6) [18]:

$$
\begin{aligned}
\sigma_{w}^{2} & =\frac{1}{a^{2} l^{2}}\left(\boldsymbol{\Psi}^{T} \boldsymbol{\Psi}\right)_{11}^{-1} \cdot \sigma^{2} \\
& =\frac{1}{a^{2} l^{2}}\left(\boldsymbol{\Psi}^{T} \boldsymbol{\Psi}\right)_{11}^{-1} N \cdot S_{y y} \cdot R
\end{aligned}
$$

$\left(\boldsymbol{\Psi}^{T} \boldsymbol{\Psi}\right)_{11}^{-1}$ is the left top element of the inverse matrix of $\boldsymbol{\Psi}^{T} \boldsymbol{\Psi}$. As clearly can be seen, $\sigma_{w}^{2}$ (unit: $\mathrm{ppm}^{2}$ ) is a function of $S_{y y}$ (unit: $1 / \mathrm{Hz}$ ), $N$ (unit: 1 ), $R$ (unit: $\mathrm{Hz}$ ), $a$ (unit: 1/ppm $\mathrm{m}$ ), optical path length $l$ (unit: $\mathrm{m}$ ) and the vectors $\boldsymbol{\psi}_{i}$. By scanning the wavelength nonlinearly or modifying the WMS modulation index $m$ or varying the scanned wavelength range, the spectral components $\psi_{i}$ change, which results in the variation of $\left(\boldsymbol{\Psi}^{T} \boldsymbol{\Psi}\right)_{11}^{-1} N$ and possibly lower values of $\sigma_{w}^{2}$. Note that $\left(\boldsymbol{\Psi}^{T} \boldsymbol{\Psi}\right)_{11}^{-1} N$ is constant for $N \rightarrow \infty$, because $\left(\boldsymbol{\Psi}^{T} \boldsymbol{\Psi}\right)_{11}^{-1}$ is asymptotically inversely proportional to $N$, whereas the limiting case also represents the continuous situation without sampling.

In the following sections, the optimum wavelength scanning functions for three different models are discussed.

\subsection{Signal model I: only target gas concentration is unknown}

If the spectral baseline is known, and there is no interfering gas or the concentration and spectrum of interfering gas are known, the model is written as

$\boldsymbol{y}=\left(\boldsymbol{\psi}_{1}\right)(C l a)+\boldsymbol{\epsilon}$,

where $\boldsymbol{y}$ is the measured data with baseline and interfering gas spectrum removed.

Substituting the model in (14) gives

$\sigma_{w}^{2}=\frac{1}{a^{2} l^{2}\left|\psi_{1}\right|^{2}} \cdot S_{y y} \cdot R \cdot N$.

It is evident that (16) is minimized if $\left|\psi_{1}\right|$ is maximized. This is achieved by sampling the absorption spectrum only at its absorption maximum (peak) value. This gives

$\boldsymbol{\psi}_{1, \mathrm{opt}}=\left(\psi_{1, \max }, \ldots, \psi_{1, \max }\right)^{T}$,

which results in

$\sigma_{w, \mathrm{opt}}^{2}=\frac{1}{a^{2} l^{2} \psi_{1, \max }^{2}} \cdot S_{y y} \cdot R$.

The corresponding spectrum waveform carries the constant value $\psi_{1, \max }$, which is 1 for direct spectroscopy and 0.343 for second harmonic detection, with $m=2.2$. The modulation index $m$ is chosen for a maximum signal peak height. For this model with known baseline and known interfering gas spectrum if there is any, it is thus optimum to sample only the maximum value of the gas absorption spectrum or the WMS harmonic spectrum.

2.5 Signal model II: target gas concentration and baseline (slope, offset) are unknown

In this case the signal model is consisting of spectral components belonging to the target gas and a spectral baseline consisting of a slope and an offset. There is no interfering gas in the spectral range and no other spectral baseline components, e.g. cubic baseline or interference fringes, or they are known and subtracted from the measurement data as in $[14,15]$.

$\boldsymbol{y}=\left(\boldsymbol{\psi}_{1}\left|\boldsymbol{\psi}_{2}\right| \boldsymbol{\psi}_{3}\right)\left(\begin{array}{c}C l a \\ \text { slope } \\ \text { offset }\end{array}\right)+\boldsymbol{\epsilon}$, 
with the new baseline components for offset and slope:

$\psi_{2}=(1 \ldots 1)^{T} \quad$ (offset),

$\psi_{3}=(-1 \ldots 1)^{T} \quad$ (slope)

Note that the slope component of the spectral baseline also changes in a nonlinear fashion when the wavelength is scanned nonlinearly. Then $\sigma_{w}^{2}$ is given by (under the assumption that $\psi_{1}$ is symmetric)

$\sigma_{w}^{2}=\frac{1}{a^{2} l^{2}\left(\left|\boldsymbol{\psi}_{1}\right|^{2}-N \overline{\boldsymbol{\psi}}_{1}^{2}\right)} \cdot S_{y y} \cdot R \cdot N$.

$\bar{\psi}_{1}$ denotes the average value of the elements of vector $\psi_{1}$. Equation (22) is minimized when $\left(\left|\boldsymbol{\psi}_{1}\right|^{2}-N \overline{\boldsymbol{\psi}}_{1}^{2}\right)$ is maximized. From optimization theory it follows that this requires sampling the minimum and maximum points of the spectrum only. Knowing this, it follows by a simple calculation that the optimum is achieved by sampling the maximum and minimum of the spectrum equally often and in a symmetrical manner as required by the assumption

$\psi_{1, \mathrm{opt}}=(\underbrace{\psi_{1, \min } \cdots}_{N / 4} \underbrace{\psi_{1, \max } \cdots}_{N / 2} \underbrace{\psi_{1, \min \cdots} \cdots}_{N / 4})^{T}$.

The minimized noise variance then has the expression

$\sigma_{w, \text { opt }}^{2}=\frac{4}{a^{2} l^{2}\left(\psi_{1, \max }-\psi_{1, \min }\right)^{2}} \cdot S_{y y} \cdot R$.

For direct spectroscopy we have $\psi_{1, \max }=1$ and $\psi_{1, \min } \approx 0$. For second harmonic detection, the peak to peak amplitude of the second harmonic spectrum is maximum when $m$ is set to 3 [20], which gives $\psi_{1, \max }=0.33$ and $\psi_{1, \min } \approx-0.22$. The optimum spectrum waveforms for target gas $\psi_{1}$ and the wavelength scanning function are shown in Fig. 6.

\subsection{Signal model III: target gas, baseline, interfering gases} and etalon fringes are unknown

In this section, an interfering gas spectrum and etalon fringes are also included in the model. A parabolic/cubic spectral baseline or polynomial arbitrary order can also be added to the model. Etalon fringes caused by interfering of light consist of a periodic sinusoidal structure in the measured spectrum; this structure can limit the sensitivity of a diode laser measurement for trace gas concentration measurements. Most of the etalon fringes are caused by parallel reflecting surfaces, between which the light is reflected back and forth several times. The reflected beams interfere at the photodetector constructively or destructively, causing the resulting intensity to be wavelength dependent. The period length of the sinusoidal structure depends on the distance between the two optical surfaces. If the distance is known,
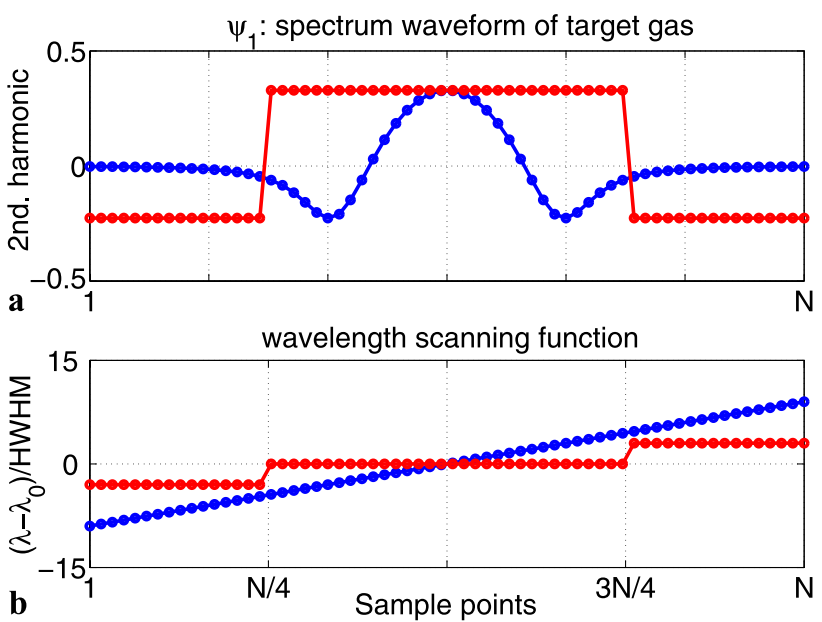

Fig. 6 The scanning waveform (second harmonic spectrum) for target gas (a) and the wavelength scanning function (b) for model II (including baseline, but no interfering gas). Traditional linear scanning (blue) and proposed optimum scanning (red) are both presented. The $y$-axis of (b) is the wavelength, which is relative to the line center $\lambda_{0}$ normalized with the HWHM of the gas absorption line

the etalon fringes can be included in the linear model, with amplitude and phase of the sinusoidal structure being fitted. This modeled spectral background consists of a linear baseline and interfering fringes with certain known period length. The amplitude of the linear baseline and amplitude and phase of the fringes are fitted continuously, so a timedependent baseline change is handled. As an extension, the fringes could be modeled not only as a single sinusoid, but as many shifted and partially overlapping finite length sinusoids. This would even allow for fitting a fringe with varying phase and amplitude during a single spectrum scan. However, simulations have shown that the optimum scanning waveform for this extended model is very similar to the simplified case used here. The model is the following:

$\boldsymbol{y}=\left(\boldsymbol{\psi}_{1}\left|\boldsymbol{\psi}_{2}\right| \boldsymbol{\psi}_{3}\left|\boldsymbol{\psi}_{4}\right| \boldsymbol{\psi}_{5} \mid \boldsymbol{\psi}_{6}\right)\left(\begin{array}{c}C l a \\ \text { slope } \\ \text { offset } \\ C_{2} l \\ A \\ B\end{array}\right)+\epsilon$,

with $\psi_{4}$ being the interfering gas spectrum and $C_{2}$ the concentration of the interfering gas. $\psi_{5}$ and $\psi_{6}$ are the components for the etalon fringes with unknown phase:

$\psi_{5}(\lambda)=\sin \left(2 \pi\left(\lambda-\lambda_{0}\right) / \Delta \lambda\right)^{T}$,

$\psi_{6}(\lambda)=\cos \left(2 \pi\left(\lambda-\lambda_{0}\right) / \Delta \lambda\right)^{T}$,

and $\Delta \lambda$ is the period length of the etalon fringes in wavelength, which is derived from the length of the etalon $L . \lambda_{0}$ 
Fig. 7 Example of simulated spectral compositions $\boldsymbol{\psi}_{i}$ in model III: $\psi_{1}$ is the second harmonic spectrum of $\mathrm{CO}$ at $2.365 \mu \mathrm{m}$ (a) with interfering gas methane $\psi_{4}$ (second harmonic spectrum of 20 methane lines) (c); $\psi_{2}$ and $\psi_{3}$ (b) are the spectral baseline: offset and slope; $\psi_{5}$ and $\psi_{6}(\mathrm{~d})$ are the sampled sine and cosine components forming the etalon fringes
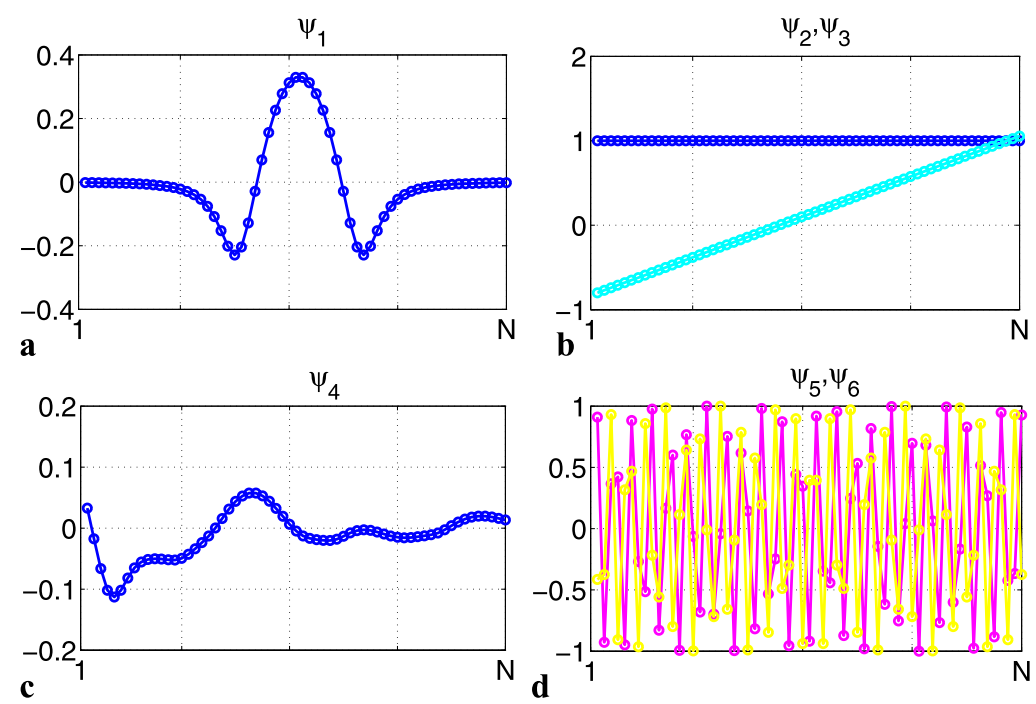

is the central wavelength of the laser light. We have

$$
\Delta \lambda=\frac{\lambda_{0}^{2}}{2 n L} .
$$

By fitting the parameters $A$ and $B$, the amplitude and phase of the sinusoidal structure are adjusted.

$\sigma_{w}^{2}$ can be only minimized numerically. The optimization problem is

$\operatorname{minimize}\left(\boldsymbol{\Psi}^{T} \boldsymbol{\Psi}\right)_{11}^{-1}$

subject to $\quad \lambda_{\min } \leq \lambda_{1} \leq \lambda_{2} \leq \cdots \leq \lambda_{N} \leq \lambda_{\max }$,

where the columns of the $\boldsymbol{\Psi}$ matrix $\boldsymbol{\psi}_{i}$ are dependent on the sampled wavelength $\lambda_{j}$. This algorithm for the constraint optimization problem [21] is described in [22, 23], and implemented in the MATLAB function 'fmincon'. We demonstrate the numerical optimization for $\mathrm{CO}$ detection at $2.365 \mu \mathrm{m}$ with the interfering gas methane. For this case, the spectral compositions $\psi_{i}$ are shown in Fig. 7. The results obtained are shown in Figs. 8 and 9 for direct spectroscopy and second harmonic detection, respectively. It is remarkable that also in the numerically optimized case the optimum wavelength scanning function has a cascade structure. The maximum is more often sampled than in the linear scanning case. The algorithm determined the modulation index $m$ to be 3, so the peak to peak amplitude of the second harmonic spectrum is maximized as in model II. On the other hand, the transitions from minimum to maximum are also sampled to obtain the information of the methane spectrum and to average over one period of the etalon fringes.

\subsubsection{Comparison between direct spectroscopy and WMS}

The sensor sensitivity $\sigma_{w}$ is multiplied with $a l / \sqrt{S_{y y} R}$ and the values are listed for comparison of linear scanning and
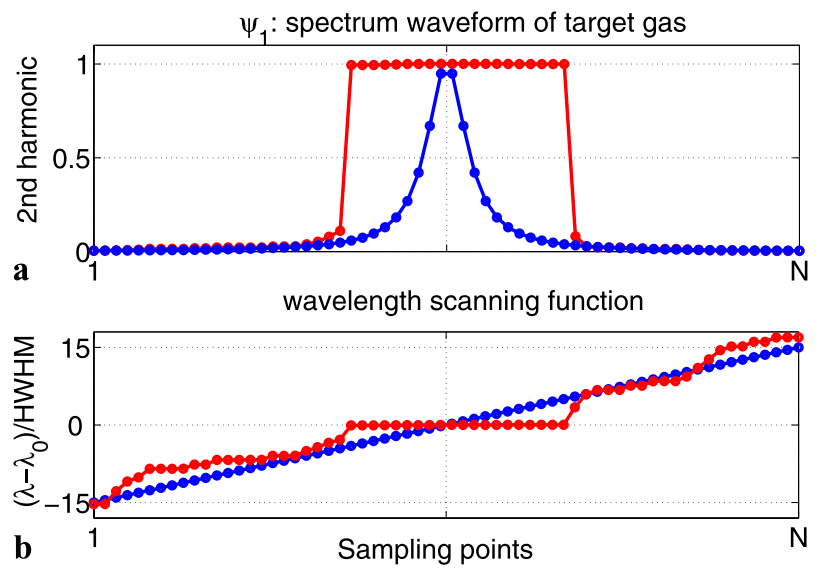

Fig. 8 (a) The spectrum waveform (direct absorption spectrum) for target gas CO and (b) the wavelength scanning function for model III. Traditional linear scanning (blue) and proposed optimum scanning (red) are both presented
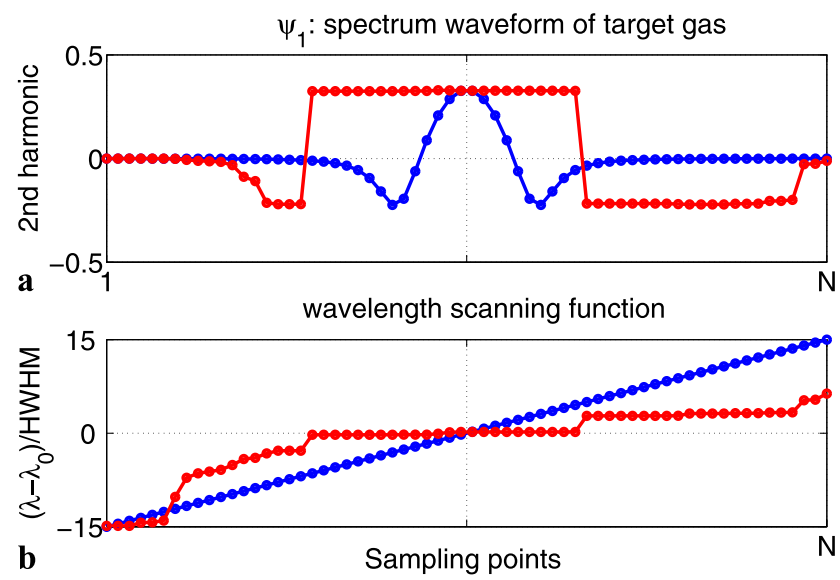

Fig. 9 (a) The spectrum waveform (second harmonic spectrum) for target gas CO and (b) the wavelength scanning function for model III. Traditional linear scanning (blue) and proposed optimum scanning (red) are both presented 
Table 1 List of $\frac{\sigma_{w} a l}{\sqrt{S_{y y} R}}$ values for the comparison of linear scanning and nonlinear wavelength scanning applying direct spectroscopy or WMS. Lower values are better

\begin{tabular}{|c|c|c|c|c|}
\hline \multirow{2}{*}{$\frac{\sigma_{w} a l}{\sqrt{S_{y y} R}}=\sqrt{\left(\boldsymbol{\Psi}^{T} \boldsymbol{\Psi}\right)_{11}^{-1} N}$} & \multicolumn{2}{|l|}{ Direct spectroscopy } & \multicolumn{2}{|c|}{ WMS (second harmonic) } \\
\hline & $\begin{array}{l}\text { Optimum nonlinear } \\
\text { scanning }\end{array}$ & $\begin{array}{l}\text { Linear scanning } \\
\text { (typical width) }^{\mathrm{a}}\end{array}$ & $\begin{array}{l}\text { Optimum nonlinear } \\
\text { scanning }\end{array}$ & $\begin{array}{l}\text { Linear scanning } \\
\text { (typical width) }^{\mathrm{a}}\end{array}$ \\
\hline I & 1 & 2.8 & $2.9^{\mathrm{b}}$ & 5.9 \\
\hline II & 2 & 3.6 & $3.6^{\mathrm{c}}$ & 5.9 \\
\hline III & 2.2 & 4.2 & $4.2^{\mathrm{c}}$ & 7.6 \\
\hline
\end{tabular}

${ }^{a}$ The width of the linear wavelength scanning is chosen as $12 \times$ HWHM of the gas absorption line for models I and II, and $20 \times$ HWHM of the gas absorption line for model III

${ }^{\mathrm{b}}$ Modulation index adjusted to maximize spectrum waveform peak $\left(m_{\mathrm{opt}} \approx 2.2\right)$

${ }^{\mathrm{c}}$ Modulation index adjusted to maximize spectrum waveform amplitude (peak to peak) $\left(m_{\mathrm{opt}} \approx 3\right)$

nonlinear wavelength scanning applying direct spectroscopy or WMS (Table 1). This value is independent of the absorption line strength (given by $a$ ), optical path length $l$, sensor time resolution $R$ and noise power density on measurement data $S_{y y}$. The lower values are better. Different models represent different levels of available a priori information. For model I (Sect. 2.4) the spectral baseline is known, for model II (Sect. 2.5) it is unknown and model III (Sect. 2.6) additionally handles interfering gases and interference fringes.

The width of the linear wavelength scanning is chosen as $12 \times$ HWHM of the gas absorption line for models I and II, and $20 \times \mathrm{HWHM}$ of the gas absorption line for model III because of the involvement of interfering gas. According to Table 1 , the standard deviation of the concentration noise/sensor detection sensitivity can be improved by a factor $\sim 2$ by applying nonlinear wavelength scanning instead of linear scanning for direct spectroscopy and WMS. Furthermore, an additional factor of $\sim 2$ lower standard deviation can be achieved theoretically if direct spectroscopy instead of second harmonic detection is applied.

With the values given in Table 1, we can also calculate the expected detection sensitivity of a sensor system. For example, assume a sensor with a measurement rate of $10 \mathrm{~Hz}$, and a high enough light intensity at the detector so that the laser noise is dominating. Additionally, we assume a laser relative intensity noise (RIN) of $-130 \mathrm{~dB} / \mathrm{Hz}$ and therefore obtain $S_{y y}=\mathrm{RIN}=10^{-13}$. The CO line at $2.3655 \mu \mathrm{m}$ has an absorption coefficient $a=3.5714 \times 10^{-5} \mathrm{ppm} \mathrm{m}^{-1}$ at ambient conditions. With an optical path length of about $10 \mathrm{~cm}$ the standard deviation of the estimated concentration value is according to the table (model III; WMS) expected to be $2.1 \mathrm{ppm}$ for linear scanning, which is in good agreement with the experimental data (standard deviation: 2-3 ppm) $[24,26]$. This performance can theoretically be improved by a factor of 1.8, if nonlinear wavelength scanning with second harmonic detection is applied, or by a factor of 3.3 using direct spectroscopy with optimum nonlinear wavelength scanning.

\subsection{Nonlinear model}

The theory for the linear model is applicable for the general nonlinear curve-fit model by linearizing it at first. The nonlinear model contains nonlinear parameters, e.g. gas pressure, gas temperature or wavelength offset. To explain this procedure, we analyze the simplest case that the model contains two parameters: one is the gas concentration $C$; the other is a nonlinear parameter $p$ (e.g. gas pressure or a wavelength shift). By applying Taylor series, the model is linearized around $\left(C_{0}, p_{0}\right)$ to

$$
\begin{aligned}
\boldsymbol{y}= & \boldsymbol{f}(C, p)+\epsilon \\
\approx & \boldsymbol{f}\left(C_{0}, p_{0}\right)+\frac{\partial \boldsymbol{f}\left(C_{0}, p_{0}\right)}{\partial C}\left(C-C_{0}\right) \\
& +\frac{\partial \boldsymbol{f}\left(C_{0}, p_{0}\right)}{\partial p}\left(p-p_{0}\right)+\epsilon \\
= & \frac{\partial \boldsymbol{f}\left(C_{0}, p_{0}\right)}{\partial C} C+\frac{\partial \boldsymbol{f}\left(C_{0}, p_{0}\right)}{\partial p}\left(p-p_{0}\right)+\epsilon \\
= & \underbrace{\left(\frac{\partial \boldsymbol{f}\left(C_{0}, p_{0}\right)}{\partial C} \mid \frac{\partial \boldsymbol{f}\left(C_{0}, p_{0}\right)}{\partial p}\right)}_{\boldsymbol{\Psi}}\left(\begin{array}{c}
C \\
p-p_{0}
\end{array}\right)+\epsilon,
\end{aligned}
$$

where $C_{0}$ and $p_{0}$ should be as close as possible to the real values of $C$ and $p$.

\section{Conclusion and discussion}

In summary, the theoretical investigation gives the optimum method to scan the laser wavelength to minimize the influence of white noise on the determined concentration values. For this, the gas absorption spectrum is probed with an optimized wavelength scanning function which enables a more efficient curve fit than for traditional linear wavelength scanning. The wavelength scanning function of the laser 
current/wavelength and the modulation index-if WMS is applied-are determined to be optimum, in the sense that the variance of the estimated concentration noise is minimized. This optimum scanning function depends on the signal model used in curve fitting. Several models including interfering gas spectra and etalon fringes are examined. While for the simple models, analytical solutions were found, for the more comprehensive cases like models including interfering gas components and etalon fringes the equation (14) were handled numerically. Compared to the gas absorption spectrum recorded by ramping the wavelength linearly, the optimum spectrum waveforms have a cascade structure, which means that the optimum scanning only samples important points of the spectrum. When the spectral baseline is unknown, the modulation index $m$ for WMS is optimally chosen to maximize the peak to peak value of the signal $(m=3)$, not the maximum value $(m=2.2)$. The new method theoretically enables a factor of $\sim 2$ improvement on detection sensitivity of the estimated concentration compared to conventional linear wavelength scanning with typical scanning width. Furthermore, direct spectroscopy is superior to second harmonic detection, because the concentration noise can be an additional factor of $\sim 2$ lower.

To use this method, knowledge of the sampled wavelength and the laser current-to-wavelength relationship is essential. For WMS it is not obvious which wavelengths correspond to the minima of the curve, because their positions are also dependent on the modulation index $m$. To obtain the laser current-to-wavelength relationship, a wavelength calibration with a reference measurement can be carried out. This can be done with a separate reference cell or integrated reference cell filled with reference gas $[24,26]$ or a reference etalon [25]. A remaining open question is whether the nonlinear scanning may increase the influence of drifts and time-varying fringes compared to linear scanning. Unfortunately, this cannot be answered by this theory. It has to be examined by experiments. The result is partly confirmed by the observed SNR improvement by using jump scanning in [12] and the used formula (14) for the SNR after the curve fit is verified in [26]. There the computed maximized SNR by optimizing the scanning width of a spectrum is in good agreement with the experimental results. In addition to the 'jump scanning' experimental paper [12], this paper tells the optimum positions where the jumps have to occur and the obtained gain in signal-to-noise ratio.

\section{References}

1. J. Reid, D. Labrie, Appl. Phys. B, Lasers Opt. 26, 203 (1981)

2. A. O'Keefe, Chem. Phys. Lett. 293, 331 (1998)

3. A. O'Keefe, J.J. Scherer, J.B. Paul, Chem. Phys. Lett. 307, 343 (1999)

4. C.B. Carlisle, D.E. Cooper, Opt. Lett. 14, 1306 (1989)

5. D.M. Sonnenfroh, W.T. Rawlins, M.G. Allen, C. Gmachl, F. Capasso, A.L. Hutchinson, D.L. Sivco, J.N. Baillargeon, A.Y. Cho, Appl. Opt. 40, 812 (2001)

6. R.E. Kalman, Trans. ASME, J. Basic Eng. 82, 35 (1960)

7. H. Riris, C.B. Carlisle, R.E. Warren, Appl. Opt. 33, 5506 (1994)

8. D. Leleux, R. Claps, W. Chen, F. Tittel, T. Harman, Appl. Phys. B, Lasers Opt. 74, 85 (2002)

9. N. Wiener, Extrapolation, Interpolation, and Smoothing of Stationary Time Series (Wiley, New York, 1949)

10. H. Riris, C.B. Carlisle, R.E. Warren, D.E. Cooper, R.U. Martinelli, R.J. Menna, P.K. York, D.Z. Garbuzov, H. Lee, J.H. Abeles, N. Morris, J.C. Connolly, S.Y. Narayan, Spectrochim. Acta Part A, Mol. Biomol. Spectrosc. 52, 843 (1996)

11. M. Sigrist, R. Bartlome, D. Marinov, J. Rey, D. Vogler, H. Wächter, Appl. Phys. B 90, 289 (2008)

12. A. Fried, B. Henry, J.R. Drummond, Appl. Opt. 32, 821 (1993)

13. P. Werle, R. Muecke, F. Slemr, Appl. Phys. B 57, 131 (1993)

14. A. Fried, B. Henry, B. Wert, S. Sewell, J. Drummond, Appl. Phys. B, Lasers Opt. 67, 317 (1998)

15. P. Werle, B. Scheumann, J. Schandl, Opt. Eng. 33, 3093 (1994)

16. P. Kluczynski, O. Axner, Appl. Opt. 38, 5803 (1999)

17. D. Bomse, D. Kane, Appl. Phys. B, Lasers Opt. 85, 461 (2006)

18. C. Rao, H. Toutenburg, Linear Models: Least Squares and Alternatives (Springer, Berlin, 1995)

19. A. Hangauer, J. Chen, M.-C. Amann, Appl. Phys. B 90, 249 (2008)

20. S. Schilt, L. Thévenaz, P. Robert, Appl. Opt. 42, 6728 (2003)

21. R. Dechter, Constraint Processing (Morgan Kaufmann, San Mateo, 2003)

22. S. Han, J. Optim. Theory Appl. 22, 297 (1977)

23. R. Fletcher, M. Powell, Comput. J. 6, 163 (1963)

24. J. Chen, A. Hangauer, R. Strzoda, M. Ortsiefer, M. Fleischer, M.C. Amann, Compact carbon monoxide sensor using a continuously tunable $2.3 \mu \mathrm{m}$ single-mode VCSEL, in Proc. 21st Annu. Meet. IEEE Lasers and Electro-Optics Society, vol. 9 (2008) pp. 721722

25. D.D. Nelson, J.B. McManus, S.C. Herndon, M.S. Zahniser, B. Tuzson, L. Emmenegger, Appl. Phys. B, Lasers Opt. 90, 301 (2008)

26. J. Chen, A. Hangauer, R. Strzoda, M.C. Amann, VCSEL-based calibration-free carbon monoxide sensor at $2.3 \mu \mathrm{m}$ with in-line reference cell. Appl. Phys. B, Lasers Opt. (2010, to be published). doi:10.1007/s00340-010-4011-0 